\section{Endoscopic ultrasound diagnosis of an ERCP-related portobiliary fistula}

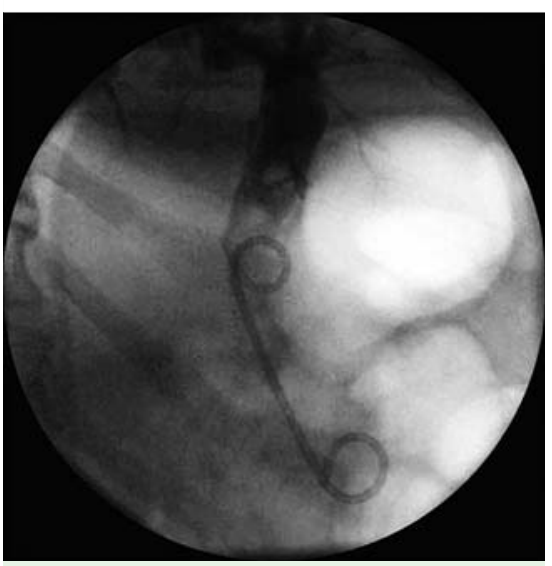

Fig. 1 Endoscopic retrograde cholangiopancreatography (ERCP) view showing a deployed pigtail stent.

A 69-year-old man, who had undergone a laparoscopic cholecystectomy 5 years previously, presented with episodic right upper quadrant pain accompanied by vomiting and sweating. Computed tomography (CT) showed two stones in a mildly dilated common bile duct (CBD). At endoscopic retrograde cholangiopancreatography (ERCP), wire-guided biliary cannulation was rendered challenging by a large periampullary diverticulum but was eventually achieved, revealing two stones in the distal CBD. A small biliary sphincterotomy was undertaken that was fol-

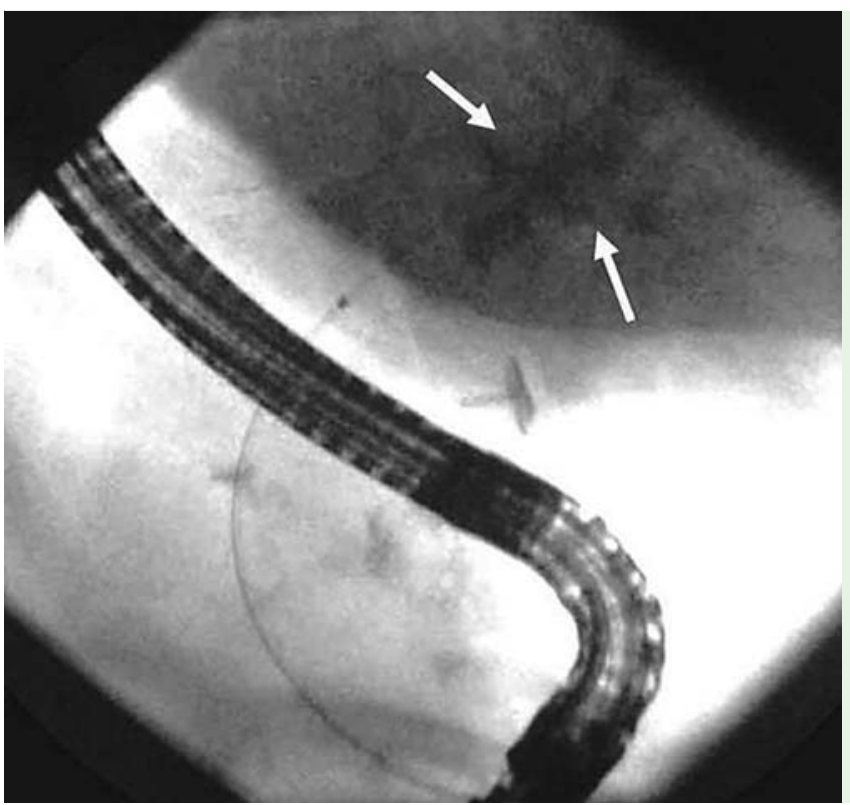

lowed by a small, self-limiting bleed. An attempt to extract the stones using a balloon was unsuccessful owing to increasing patient restlessness and was complicated by dislodgment of the cannulation wire from the CBD. Biliary recannulation proved difficult and the procedure was terminated after deployment of a 4-cm, 7-Fr, double pigtail, plastic biliary stent $(\bullet$ Fig. 1). The patient experienced troublesome abdominal pain and sweating after the procedure, requiring a brief hospital admission 3 days later. However, physical examination and a plain X-ray were unrevealing, laboratory investigation showed only a C-reactive protein of $61 \mathrm{mg} / \mathrm{L}$, and the pain settled with conservative management. The patient underwent a further ERCP 5 weeks later during which removal of the biliary stent was complicated by a modest, spontaneously resolving bleed. Wire-guided cannulation at the previous sphincterotomy site revealed only faint, rapidly dissipating, biliary-like opacification despite several contrast injections ( $\bullet$ Fig.2). The procedure was abandoned and, although the patient remained entirely asymptomatic thereafter, an urgent CT scan was performed. This disclosed gas shadowing in the portal vein extending to its peripheral intrahepatic branches, a thrombus in the main portal vein, and a small amount of peri-

Fig. 2 Endoscopic retrograde cholangiopancreatography (ERCP) view showing portal vein opacification (arrows). pancreatic and periportal free gas in keeping with a localized perforation ( $\$$ Fig. 3 ). A pancreaticobiliary endoscopic ultrasound (EUS) performed 8 days later demonstrated evidence of a communication between the portal vein and $\mathrm{CBD}$, the two structures being inseparable at the level of the mid-duct in keeping with a portobiliary fistula ( Video 1 ). However, no blood flow could be detected within the CBD, possibly due to the presence of a thrombus, albeit nonocclusive, within the portal vein. In addition, multiple CBD stones were seen (not shown). A CT scan carried out 2 months later confirmed spontaneous resolution of all portal vein abnormalities and extraluminal free gas. Complete biliary clearance was accomplished at subsequent ERCP ( $\bullet$ Fig. 4$)$.

Portal vein opacification is an exceedingly rare event during ERCP with an estimated incidence of 1 in 6000-8000 [1]. First reported by Huibregtse et al. in 1988 [2], it has since been described in several case reports, mostly, but not exclusively, in the setting of attempted biliary cannulation following needle-knife precut or biliary sphincterotomy [3]. However, to our knowledge, the existence of a portobiliary fistula per se has hitherto been demonstrated at ERCP in only three cases, two of which having been thought to be secondary to multiple liver abscesses [4,5] and the third occurring in the setting of a presumed portal vein tumor thrombus [6]. The case reported here represents the first to be documented by EUS. Other, more common, causes of portobiliary fistulas include percutaneous transhepatic cholangiography, choledocholithiasis, biliary surgery, trauma, and percutaneous liver biopsy [6]. Demonstration of a portopancreatic fistula at ERCP has also been described in the setting of pancreatic pseudocyst or malignancy [7]. The more general finding of hepatic portal venous gas on plain X-rays, CT or doppler ultrasound has a wide differential diagnosis including, in addition to portobiliary fistula, necrotizing enterocolitis (in neonates), mesenteric ischemia, intra-abdominal sepsis (particularly diverticulitis), acute gastric dilatation, gastric emphysema, inflammatory

\section{Video 1}

Linear pancreatico-biliary endoscopic ultrasound scan showing a portobiliary fistula (PBF) and a nonocclusive portal vein (PV) thrombus. The common bile duct (CBD), main pancreatic duct (PD), and head of pancreas are also shown. 


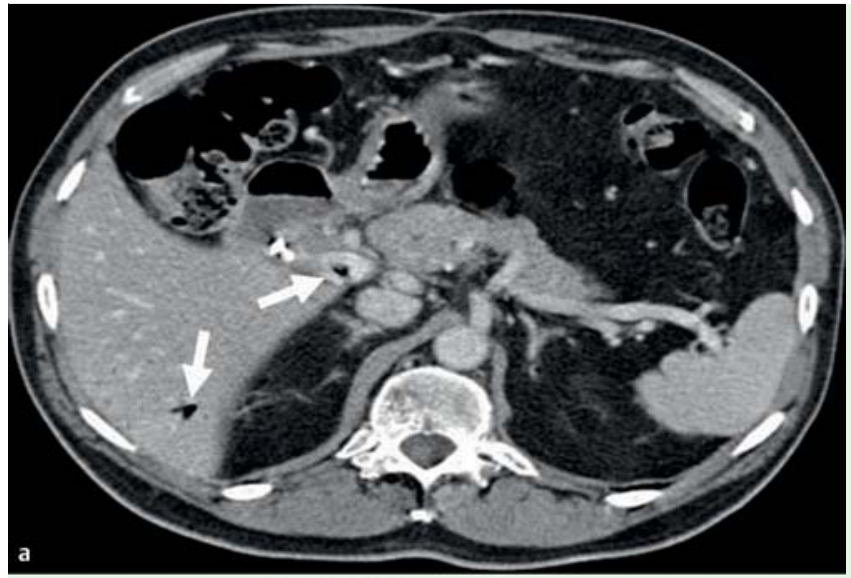

Fig. 3 Computed tomography (CT) scan showing: $\mathbf{a}$ air in the main portal vein and a peripheral intrahepatic branch (arrow); b main portal vein thrombus (arrow); and c free periduodenal gas (arrow).
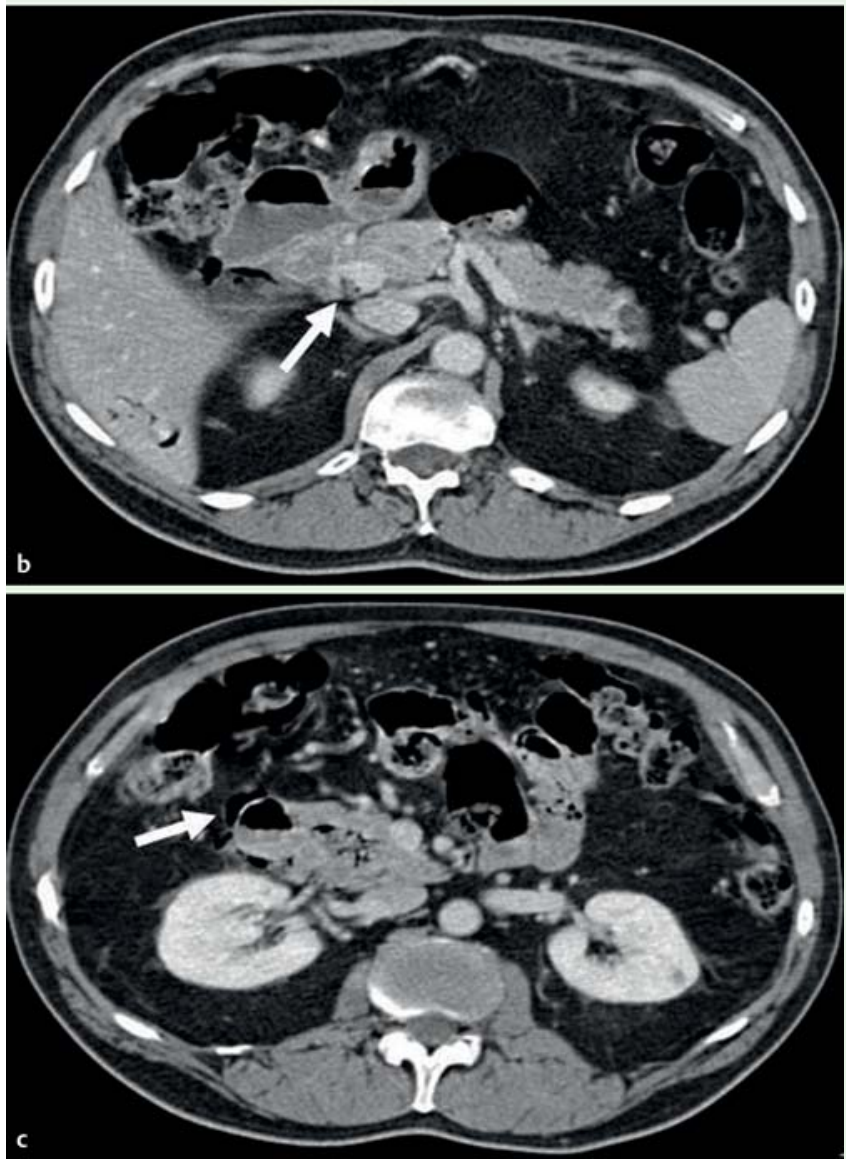

bowel disease, liver transplantation, chemotherapy, and a variety of other endoscopic procedures [8].

Clinically, hemobilia and right upper quadrant pain are the most common manifestations of portobiliary fistulas [6] although previously reported cases of portal vein opacification at ERCP, with or without proven portobiliary fistulas, have all been associated with surprisingly little bleeding [9]. Other potential clinical features include those of sepsis, portal vein thrombosis, and air embolism.

Management may entail surgical repair, thrombin injection, coil occlusion, simple or balloon tamponade, or transhepatic biliary or portal vein stenting $[10,11]$. Typically, however, portal vein opacification in the setting of ERCP tends to have a favorable prognosis in the absence of further intervention [9] with spontaneous clearance of hepatic portal venous gas within as little as 4 hours [12], although this may merely reflect reporting bias. Indeed, a single fatality related to air embolism as a consequence of overlooking the diagnosis has been described [13]. Thus, immediate termination of the procedure is recommended and, to this end, prompt recognition of portal vein opacification as a radiographic pattern that could closely mimic that of biliary underfilling (with

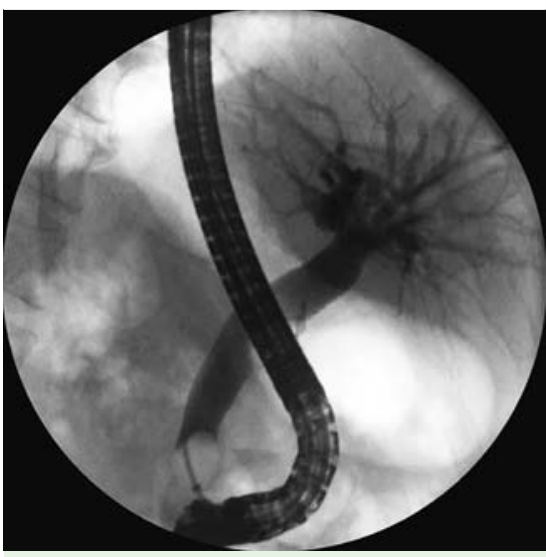

Fig. 4 Occlusion cholangiogram confirming ductal clearance.

striking similarities between the two structures in location, orientation, and branching pattern) is imperative; perseverance in the event of misinterpretation may lead to potentially deleterious therapeutic misadventures such as transduodenal portal vein diathermy or stenting. Aspiration of blood rather than bile upon cannulation may facilitate a prompt diagnosis.

Endoscopy_UCTN_Code_CPL_1AK_2AI

Competing interests: None

M. F. Dawwas' ${ }^{1}$ K. W. Oppong ${ }^{1}$,

S. K. John ${ }^{1}$, A. E. Vallance', J. J. French', J. Scott ${ }^{2}$, M. K. Nayar ${ }^{1}$

${ }^{1}$ Hepato-Pancreato-Biliary Centre,

Freeman Hospital, Newcastle upon Tyne, United Kingdom

2 Department of Radiology, Freeman Hospital, Newcastle upon Tyne, United Kingdom

\section{References}

1 Siegel JH, Ben-Zvi JS, Yatto RP. Portal vein filling during ERCP. Gastrointest Endosc 1993; 39: $471-472$

2 Huibregtse K, Gish R, Tytgat GN. A frightening event during endoscopic papillotomy. Gastrointest Endosc 1988; 34: 67-68

3 Kalaitzakis E, Stern N, Sturgess R. Portal vein cannulation: an uncommon complication of endoscopic retrograde cholangiopancreatography. World J Gastroenterol 2011; 17: 5131-5132

4 Skelly MM, Ryder SD. Images in hepatology. Liver abscesses with bilioportal fistula. J Hepatol 1999; 31: 365

5 O'Connor AR, Curtis H, Dunn WK. Portal venous opacification at ERCP in association with multiple hepatic abscesses and portal vein thrombosis. Clin Radiol 2000; 55: 980-982 
6 Quinn C, Johnston SD. Portobiliary fistula at ERCP. Gastrointest Endosc 2007; 65: $175-$ 177

7 Lum C, Cho KC, Scholl DG et al. Portal vein opacification during ERCP in patients with pancreatitis. Abdom Imaging 1998; 23: $81-83$

8 Abboud B, El Hachem J, Yazbeck T et al. Hepatic portal venous gas: physiopathology, etiology, prognosis and treatment. World J Gastroenterol 2009; 15: 3585 - 3590

9 Furuzono M, Hirata $N$, Saitou J et al. A rare complication during ERCP and sphincterotomy: placement of an endoscopic nasobiliary drainage tube in the portal vein. Gastrointest Endosc 2009; 70: 588 - 590
10 Peynircioglu B, Cwikiel W. Utility of stentgrafts in treatment of porto-biliary fistula. Cardiovasc Intervent Radiol 2006; 29: 1156-1159

11 Kasahara M, Sakamoto S, Fukuda A et al. Posttransplant bilioportal fistula with portal vein thrombosis: a case report. Transplant Proc 2010; 42: 3862 - 3864

12 Simmons TC. Hepatic portal venous gas due to endoscopic spincterotomy. Am J Gastroenterol 1988; 83: 326-328

13 Kennedy C, Larvin M, Linsell J. Fatal hepatic air embolism following ERCP. Gastrointest Endosc 1997; 45: 187-188

\section{Bibliography}

DoI http://dx.doi.org/

10.1055/s-0033-1344065

Endoscopy 2013; 45: E214-E216

(c) Georg Thieme Verlag KG

Stuttgart · New York

ISSN 0013-726X

\section{Corresponding author}

\section{F. Dawwas}

Hepato-Pancreato-Biliary Centre

Freeman Hospital

Freeman Road

Newcastle upon Tyne

NE7 7DN

United Kingdom

Fax: +44-191-2231155

drdawwas@gmail.com 